\title{
Discours
}

Revue de linguistique, psycholinguistique et

informatique. A journal of linguistics, psycholinguistics and computational linguistics

9| 2011

Varia

\section{Étude comparée du fonctionnement des parenthèses et des tirets}

\section{Guillaume François}

\section{(2) OpenEdition \\ Journals}

Édition électronique

URL : http://journals.openedition.org/discours/8542

DOI : $10.4000 /$ discours. 8542

ISSN : 1963-1723

Éditeur :

Laboratoire LATTICE, Presses universitaires de Caen

\section{Référence électronique}

Guillaume Francois, «Étude comparée du fonctionnement des parenthèses et des tirets », Discours

[En ligne], 9 | 2011, mis en ligne le 20 décembre 2011, consulté le 01 mai 2019. URL : http:// journals.openedition.org/discours/8542 ; DOI : 10.4000/discours.8542

\section{(c) (i) (9)}

Discours est mis à disposition selon les termes de la licence Creative Commons Attribution - Pas d'Utilisation Commerciale - Pas de Modification 4.0 International. 

Revue de linguistique, psycholinguistique et informatique

\section{Étude comparée du fonctionnement des parenthèses et des tirets}

Guillaume François

Guillaume François, «Étude comparée du fonctionnement des parenthèses et des tirets», Discours [En ligne], 9 | 2011, mis en ligne le 20 décembre 2011.

URL: http://discours.revues.org/8542. Consulté le 20 décembre 2011.

Titre du numéro: Varia

Coordination: Anne Le Draoulec et Josette Rebeyrolle

revues.org CENTRE POUR L'ÉDITION ÉLECTRONIQUE OUVE
CENTRE For OPEN ELECTRONIC PUBLISHING

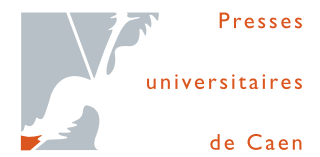

de Caen 



\title{
Étude comparée du fonctionnement des parenthèses et des tirets
}

Guillaume François

\begin{abstract}
Nous nous interrogeons ici sur l'équivalence fonctionnelle des parenthèses et du tiret parenthétique. Les deux signes marquent tous deux «l'opération de décrochement typographique» (Pétillon-Boucheron, 2003). Cependant, notre étude de corpus montre clairement que leurs usages ne sont pas les mêmes. Alors que la parenthèse semble toujours pouvoir se substituer au tiret, l'inverse n'est pas vrai. La parenthèse a des usages propres tant au niveau énonciatif (marquage du discours autre) que sémantique ou syntaxique (marquage d'éléments inférieurs au mot, supérieurs ou égaux à la phrase). Par ailleurs, les parenthèses sont toujours doubles alors que le tiret fermant «s'efface» devant une ponctuation forte. Nous tentons d'expliquer cette différence de fonctionnement par une différence de position dans le système de la ponctuation. Selon nous, les tirets sont des signes de phrase (dont la portée est strictement inférieure à celle-ci) alors que les parenthèses sont des signes de l'énonciation, autrement dit des signes qui peuvent se «balader» à tous les niveaux d'organisation textuelle (du caractère aux paragraphes).
\end{abstract}

Mots clés : ponctuation, parenthèse, tiret parenthétique, marquage métadiscursif, variantes graphiques

This study investigates the functional equivalence of brackets and dashes. Both signs seem to mark "the operation of typographic uncoupling" (Pétillon-Boucheron, 2003). Our corpus study clearly shows, however, that their uses differ. While brackets can always replace dashes, the reverse is not true. Brackets have specific uses both on the enunciative level (marking someone else's speech) and with respect to the semantics or syntax (marking items below the word, greater than or equal to the sentence). In addition, while brackets are always double, the closing dash is "erased" before a strong punctuation mark. We attempt to explain this difference in their operation by a difference in their positions in the punctuation system. We argue that dashes are sentential signs (whose scope is strictly below the sentence) while brackets are enunciation signs, i.e. signs that can range over all levels of textual organization (from characters to paragraphs).

Keywords: punctuation, brackets, parenthetic dash, metadiscursive marking, graphic variants

Lorsqu'à l'écrit, on désire signaler typographiquement qu'un élément est un ajout au discours principal, on dispose de deux signes de ponctuation distincts: les parenthèses et les tirets parenthétiques ${ }^{1}$. Leur fonctionnement énonciatif a déjà été largement étudié : on peut en retenir sommairement que ces signes sont le lieu du dédoublement de la voix de l'énonciateur, l'endroit où ce dernier décide de créer un espace graphique pour «ajouter par ailleurs» une information

1. Nous préférons cette appellation à celle usuelle de tirets doubles parce qu'il est courant qu'un seul tiret soit réalisé (voir partie 3 ci-après ou, pour une argumentation plus détaillée, Pétillon-Boucheron [2003]). 
quelconque; en ce sens Pétillon-Boucheron parle de «décrochement énonciatif ${ }^{2}$. Nous ne nous attarderons donc plus sur cet aspect.

La question restée sans réponse est celle de la différence de fonctionnement des deux signes. Si Pétillon-Boucheron (2003: 93-94) les considère comme des «variantes graphiques» d'un même signe «qu'I. Fonagy qualifierait de synonymes», la plupart des linguistes en font des signes distincts dotés d'une signification différente. On peut ainsi lire chez Doppagne (1998: 64-65):

En tant que signes d'insertion, les tirets se situent, pour leur puissance expressive, à peu près au niveau des parenthèses. Le message à insérer dans un texte, peut l'être différemment selon que l'on recourt aux parenthèses ou aux tirets. [...] [N]ous pouvons comparer les effets produits par d'autres signes que l'on pourrait charger de cette mission d'insertion. Les parenthèses [...] laisseraient la remarque dans l'ombre, auraient tendance à la minimiser. Les virgules [...] sembleraient mettre tout sur le même pied et rendraient l'expression plate et neutre. Les tirets relèvent l'expression au lieu de l'amoindrir et attirent l'attention sur la remarque qu'ils contiennent plutôt que d'inviter à la négliger.

Ou chez Anis (1988: 136-I37):

Le tiret parenthétique est assez difficile à distinguer fonctionnellement de la parenthèse: cependant sa valeur de base semble être plus d'apporter un supplément qu'un complément d'information - comme la parenthèse; l'intégration au texte est plus forte, le segment entre tirets relève du même mouvement énonciatif, participe de la signification globale de l'énoncé [...].

Drillon (1991 : 329-300) affirme au contraire que le tiret marque une rupture plus nette que la parenthèse :

Le tiret, plus encore que la parenthèse, interrompt la continuité de la phrase. Il inclut de force, pourrait-on dire, une phrase dans la phrase; elle y garde son indépendance syntaxique et/ou sémantique. Plus que la parenthèse, le tiret est extérieur à l'unité de la phrase.

Serça (20IO: IOO-IOI) estime que la question du poids sémantique est à exclure au profit du poids énonciatif:

[...] [L]a différence n'est sans doute pas à poser en termes de poids sémantique, mais en termes de poids énonciatif: les parenthèses introduiraient une rupture énonciative plus nette que celle qu'opèrent les tirets. On pourrait ainsi poser un continuum sur lequel le tiret double vient s'inscrire entre la double virgule et les parenthèses. Les tirets, qui seraient de grosses virgules, marqueraient alors un

2. Pour une discussion sur le sens des parenthèses et des tirets, on se reportera utilement à PétillonBoucheron (2003), Anis (I988: II8-II9) ou François (2006: 239 sq.). 
décalage propre au locuteur alors que les parenthèses signaleraient un changement d'instance, avec une rupture énonciative franche.

Le but de la présente contribution est d'examiner, sur la base d'une étude de corpus $^{3}$, dans quelle mesure les deux signes sont équivalents. Nous procéderons à une analyse pragmatique et sémantique puis syntaxique, avant de terminer par une étude contrastive de leurs fonctionnements typographiques et une explication des différences ainsi observées.

\section{Analyse pragmatique et sémantique}

Les parenthèses et les tirets sont, nous l'avons déjà dit, le lieu du décrochement typographique où l'énonciateur «ajoute par ailleurs». Ces ajouts du scripteur dans son propre écrit sont constitués de précisions, de commentaires métadiscursifs et de navigation intra- et intertextuelle.

\subsection{Précisions}

Avec les tirets ou les parenthèses, l'énonciateur peut préciser de maintes manières son énoncé ${ }^{4}$ :

- en précisant un ordre de grandeur:

[I] Cet effet reste néanmoins très mal cerné, à la fois sur le plan des concentrations - qui varient du simple au double, voire plus - et sur celui des mécanismes impliqués. (La recherche. L'actualité des sciences, $\mathrm{n}^{\circ} 360$, janvier 2003, p. 15$)^{5}$

3. Le corpus ici utilisé est celui de François (2006). Il se compose d'un corpus de presse (presse quotidienne [Le Monde, $14 / \mathrm{o}_{3} / 2003 ;$ Le Soir, $\mathrm{17} / \mathrm{o}_{3} / 2003$ ], presse «d'information» [Le Monde diplomatique, $\mathrm{n}^{\circ} 600$, mars 2004; Le Vif/L'Express, 17-23/03/2003 et 03-09/07/2003], presse gratuite [Metro, 27/0I/2005], presse féminine [Marie-Claire, n 6I8, février 2004; Maxi, nº 898, I2-I8/oI/2004; Gaël, nº I83-I, janvier 2004; 20 ans, $\mathrm{n}^{\circ}$ 193, octobre $2002 ;$ Femme actuelle, $\mathrm{n}^{\circ}$ I036, 02-08/08/2004], presse relatant l'actualité des «stars» [Public [édition belge], $\mathrm{n}^{\circ} 56,09-15 / 08 / 2004$ ], presse de vulgarisation scientifique [La recherche. L'actualité des sciences, $\mathrm{n}^{\circ} 360$, janvier 2003; National Geographic France, $\mathrm{n}^{\circ} 4 \mathrm{I}$, février 2003; Science et vie, $\mathrm{n}^{\circ}$ IO33 , octobre 2003; La lettre du FNRS, $\mathrm{n}^{\circ} 54$, septembre 2003], presse relative à la télévision [Télé Moustique, $\mathrm{n}^{\circ} 13,22-28 / 03 / 2003, \mathrm{n}^{\circ} 35,23-29 / 08 / 2003, \mathrm{n}^{\circ} 39,20-29 / 09 / 2003, \mathrm{n}^{\circ} 53,27 / \mathrm{I2} / 2002-02 / 01 / 2003, \mathrm{n}^{\circ} 2$, IO-I6/OI/2004, $\left.\left.\mathrm{n}^{\circ} 2 \mathrm{I}, 22-28 / 05 / 2004\right]\right)$ et d'un corpus littéraire (P. Grainville, Les forteresses noires, Paris, Seuil, 1982; J. Harpman, La plage d'Ostende, Paris, Stock, I991 ; J.-L. Outers, L'ordre du jour, Arles, Actes Sud, 1996), auxquels nous avons ajouté quelques exemples glanés au fil de nos lectures (Têtu, $\mathrm{n}^{\circ} 75$, février 2003 et $\mathrm{n}^{\circ} 58$, mai 2003; Public, $\mathrm{n}^{\circ}$ 80, 21-30/0I/2005; Elle, 20/08/2004; Le Vif/L'Express, 27/06-03/07/2003; Le Soir et Le Monde consultés en ligne; Metro Summertime, nº 609, or-05/08/2003; Télé Moustique, $\mathrm{n}^{\circ}$ 8, 24-28/02/2006; Science et vie, $\mathrm{n}^{\circ}$ I029, juin 2003; F. Cavanna, "Mignonne, allons voir si la rose», Paris, P. Belfond, I989; N. Sarraute, Enfance, Paris, Gallimard, I983; D. Pennac, Monsieur Malaussène, Paris, Gallimard, 1995) ainsi que des exemples extraits de Pétillon-Boucheron (2003).

4. La liste ci-dessous ne se veut pas exhaustive. Elle donne simplement un aperçu des usages les plus courants.

5. Les soulignements dans les exemples sont de notre fait, les autres marques typographiques sont de l'auteur, sauf indication contraire. 
[2] Bonne nouvelle pour eux: cette année, d'après le magazine «Forbes», i7 Russes seraient milliardaires (en $\$)$, contre 7 en 2002.

(Télé Moustique, $\mathrm{n}^{\circ}$ 13, 22-28/03/2003, p. 7)

- en donnant des exemples:

[3] Les appareillages orthopédiques sont devenus plus légers et plus confortables (on peut désormais porter des vêtements moulants, par exemple).

(Têtu, $\mathrm{n}^{\circ} 75$, février 2003, p. I2)

[4] Cette simplicité ajoutée à sa serviabilité - pour tout stylo commandé, il vous en donnait deux plutôt qu'un - faisait que tout le monde aimait beaucoup M. Libert.

(J.-L. Outers, L'ordre du jour, p. I9)

- en donnant certaines caractéristiques:

[5] Après le départ de Roland Mureau, parti à l'administration fédérale (Le Vif/L'Express du 9 mai 2003), c'est Peter Desmet (42 ans) qui prend, le $\mathrm{I}^{\text {er }}$ juillet prochain, les commandes de Greenpeace Belgium.

(Le Vif/L'Express, 27/06-03/07/2003, p. 9)

[6] Otto Nijenhuis - qui vient d'une maison doublement étoilée aux Pays-Bas - affiche son originalité avec panache.

(Gaël, $\mathrm{n}^{\circ}$ I83-I, janvier 2004, p. I5)

- en explicitant certains éléments :

[7] Au-delà de leur endurance physique (chacun transportait quotidiennement plus de $25 \mathrm{~kg}$ d'équipements divers, balluchons, tentes, nourritures, etc.) et de leur expertise de la montagne, nos amis Chaggas ont d'ailleurs très largement contribué au succès de l'expédition [...].

(Télé Moustique, ${ }^{\circ}$ 13, 22-28/o3/2003, p. 36)

[8] Mais je travaille aussi pour le théâtre - j'ai réalisé le corset de la robe d'Adjani dans La Dame aux camélias - et pour le cinéma. J'ai corseté Catherine Frot pour l'adaptation de Vipère au poing.

(Femme actuelle, $\mathrm{n}^{\circ}$ I036, 02-08/08/2004, p. I8)

- en exprimant une cause:

[9] Alors que, depuis quelques années, l'industrie cinématographique américaine multiplie les tournages à l'étranger (principalement pour raisons économiques), celle-ci est à présent obligée de reporter certains projets d'envergure du fait de leurs lieux de tournage, trop rapprochés du chaudron irakien.

(Télé Moustique, $\mathrm{n}^{\circ}$ 13, 22-28/03/2003, p. 8) 
[io] Et puis, je préfère donner que recevoir - j'aime bien voir le plaisir que cela fait, un peu comme en amour...

(Télé Moustique, n² 2, 10-16/or/2004, p. 5)

- une conséquence:

[II] De telles sollicitations incitent l'activité de réseaux de prélèvement et d'exportation clandestines [sic], dont font dejà les frais mygales, perroquets et dendromates, ces dernières n'étant absolument pas aussi dangereuses - et donc fascinantes! - que la rumeur le colporte.

(National Geographic France, n ${ }^{\circ}$ 4I, février 2003)

[i2] Les appareillages orthopédiques sont devenus plus légers et plus confortables (on peut désormais porter des vêtements moulants, par exemple).

(Têtu, $\mathrm{n}^{\circ} 75$, février 2003, p. I2)

- une restriction:

[13] Parce que, quand j'étais petit garçon - et bien que je n'aie jamais manqué d'amour -, j'ai remarqué que personne ne s'intéressait à moi, sauf quand je commençais à chanter.

(Télé Moustique, n², I0-16/oI/2004, p. 5)

[I4] Michael Jackson n'aurait jamais subi d'opération de chirurgie esthétique! (à part deux petites opérations du nez de rien du tout).

(Télé Moustique, n 13, 22-28/03/2003, p. 19)

- une addition:

[I5] [...] il finira par se confirmer que chaque détail de notre Univers suit effectivement des règles qui peuvent être représentées par un programme simple - et que tout ce que nous voyons finira par émerger rien qu'en faisant tourner ce programme simple. (La recherche. L'actualité des sciences, $\mathrm{n}^{\circ} 360$, janvier 2003, p. 34)

[ı] En tout cas, ce que d'aucuns, en Irak, appellent la «résistance à l'occupation» prend de l'ampleur. Sans doute, les actes antiaméricains (et antibritanniques) plafonnentils désormais à douze/quinze par jour, ce qui n'est pas négligeable, mais surtout ils se font plus précis, plus sophistiqués et mieux ciblés.

(Le Soir en ligne, consulté sur http://archives.lesoir.be/irak-plusieurs-sortes-dhommes-ou-de-groupes-armes_t-20030819-ZoNF54.html)

- des indications spatio-temporelles:

[17] Huit jours de marche et l'avant-dernière étape (avant la redescente) d'un «trek» entamé cinq jours plus tôt.

(Télé Moustique, $\mathrm{n}^{\circ}$ 13, 22-28/03/2003, p. 35) 
[18] On y retrouve - pendant les trois premiers épisodes - le personnage de Michael J Fox (Mike) [...].

(Télé Moustique, $\mathrm{n}^{\circ}$ 13, 22-28/03/2003, p. 18)

On notera cependant que seules les parenthèses sont aptes à encadrer les dates de naissance et de mort:

[19] Maurice Hagemans (Liège, I852 - Ixelles, 1917), le peintre de ce tableau paisible, a su combiner un certain réalisme avec l'atmosphère des prairies et des changements de lumière.

(Metro Summertime, $\mathrm{n}^{\circ}$ 609, oI-05/08/2003, p. 3)

Ce sont probablement des raisons de lisibilité qui expliquent ce phénomène. En effet, les deux indications sont séparées par un tiret, et encadrer la séquence entière de ce même signe la rendrait peu lisible:

[19b] *Maurice Hagemans - Liège, I852 - Ixelles, 1917-, le peintre de ce tableau paisible, a su combiner un certain réalisme avec l'atmosphère des prairies et des changements de lumière.

(Metro Summertime, no 609, oI-05/08/2003, p. 3)

Dans cette configuration, on ne voit pas, en effet, que les lieux et dates forment un bloc lui-même encadré d'une paire de signes ${ }^{6}$.

\subsection{Commentaires métadiscursifs}

Parenthèses et tirets permettent également au scripteur de commenter son propre discours ${ }^{7}$.

Ce métadiscours peut marquer la conscience qu'a le scripteur de ne pas être compris par son lecteur-cible. Cette opération pourra être constituée:

- de simples sigles:

[20] La police libanaise a indiqué que des batteries de défense anti-aérienne (DCA) étaient entrées en action dimanche contre des avions israéliens qui violaient l'espace aérien du Liban sud.

(Le Monde, $14 / 03 / 2003$, consulté sur Internet, lien indisponible)

6. Une présentation du type «- Liège, 1852 /Ixelles, $1917-»$ ne semble pas totalement impossible, mais n'est pas attestée dans notre corpus.

7. Une étude détaillée des usages métadiscursifs de nos signes est fournie par Pétillon-Boucheron (2003: 237-328). Il ne s'agit pas ici de reproduire une étude aussi fouillée, mais plus modestement de donner quelques éléments centraux qui permettent de mettre en lumière les ressemblances et dissemblances de fonctionnement des ponctuations ici étudiées. 
[2I] Récemment, le développement de nouvelles technologies d'ionisation telles que l'électronébulisation - ESI - ou la désorption laser-MALDI -, a permis d'étendre la gamme des produits analysables jusqu'aux molécules non volatiles comme les biomolécules. (La lettre du FNRS, $\mathrm{n}^{\circ}$ 54, septembre 2003, p. 15)

- de traductions:

[22] À quelques dizaines de mètres de ces innovations high-tech, les visiteurs peuvent pénétrer dans le «sprookjesbos» (le bois des contes) où des automates au charme un peu suranné mettent en scène quelques-unes des plus célèbres histoires de fées. (Le Soir en ligne, consulté sur http://archives.lesoir.be/tourisme-5-7-une-journeede-detente-ou-de_t-20030819-ZoNF5U.html)

[23] À titre d'exemple, les recherches effectuées sur un large échantillonnage de mulots sylvestres - apodemus sylvaticus - et de mulots à collier - a. flavocollis - ont permis de montrer que les régions méditerranéennes représentent pour ces espèces et d'autres étudiées ultérieurement, des zones refuges où elles ont pu survivre durant les périodes glaciaires.

(La lettre du FNRS, n ${ }^{\circ}$ 54, septembre 2003, p. 22)

- ou de véritables définitions:

[24] Parce que ce sont eux qui prennent le plus d'immunosuppresseurs (des médicaments destinés à contrôler le rejet - NDLR).

(Télé Moustique, $\mathrm{n}^{\circ} \mathrm{13}, 22-28 / 03 / 2003$, p. 38)

[25] Par exemple on n'observe pas ou peu de réparation spontanée d'un territoire cérébral qui a subi une dépopulation neuronale suite à une ischémie - dommage tissulaire par obstruction ou thrombose du vaisseau irriguant un territoire déterminé - ou qui est le site d'un processus de démyélinisation lors d'une poussée aiguë à un stade avancé d'une maladie comme la sclérose en plaques [...].

(La lettre du FNRS, $\mathrm{n}^{\circ} 54$, septembre 2003, p. 2)

De manière encore plus claire, l'énonciateur commente son propre énoncé :

- en signalant une alternance syntagmatique placée sur l'axe paradigmatique:

[26] Et l'on voit aussi (surtout) ses jambes pendantes et flasques, parfois prises de tremblements (c'est mon cas).

(Têtu, $\mathrm{n}^{\circ} 75$, février 2003, p. I2)

[27] Nous étions entrés de plain-pied dans un domaine interdit, et le monde du dehors, le monde lointain dont pour l'instant nous ne faisions pas partie, ne pourrait avoir pour nous que blâme et refus - n'avait pour nous que blâme et refus mais ne le savait pas: c'était notre tâche de le laisser dans l'ignorance.

(J. Harpman, La plage d'Ostende, p. 76) 
- en commentant ses choix lexicaux:

[28] On n'arrête pas d'entendre et de lire: quel dommage que l'Europe se soit montrée divisée sur la crise américaine (excusez-moi, je n’arrive décidément pas à appeler ça la crise irakienne).

(Télé Moustique, $\mathrm{n}^{\circ}$ 13, 22-28/03/2003, p. I4)

[29] Je crois que, s'il n'avait pas fait la sottise de m'ôter à Bruxelles, je ne l'aurais jamais trompé - j’avais encore la plume dans le mot «trompé» que je riais déjà, tant il est évident pour moi qu'avec Léopold je n’ai pas trompé Charles: autant dire qu'il l'épousait en même temps que moi!

(J. Harpman, La plage d'Ostende, p. 152-153)

- en commentant la syntaxe de l'énoncé :

[30] «Choisissez le droit à l'indifférence aux dépens du désir de différence» nous lance finalement notre nouveau conseiller, dont nous sommes les nouveaux maîtres (vous suivez?).

(Têtu, ${ }^{\circ} 58$, mai 2003, p. 4)

[3I] L'émission s'appelle - respirez un grand coup - A la recherche de la nouvelle star («on dirait du Proust!», dit Virginie).

(Télé Moustique, $\mathrm{n}^{\circ} \mathrm{13}$, 22-28/03/2003, p. 32)

Lorsque le scripteur désire signaler son attitude face à un élément (doute, étonnement, surprise, etc.), il peut placer entre parenthèses un point d'interrogation ou d'exclamation sans autre marque linguistique. Dans ce cas, seule la parenthèse a été observée:

[32] Le gagnant signe un contrat «platinium» qui lui assure notamment une meilleure promotion (!).

(Télé Moustique, $\mathrm{n}^{\circ} \mathrm{13}, 22-28 / 03 / 2003$, p. 32)

[33] Quant aux arguments spécieux par lesquels les défenseurs du point-virgule essaient de justifier son emploi : comme quoi il marquerait une halte moins «brutale» (?) que celle du point, intimerait une injonction plus nuancée, moins autoritaire, une espèce de freinage en douceur opposé au coup de patin sans réplique du point... Mouais. C'est tout simplement un aveu d'échec.

(F. Cavanna, «Mignonne, allons voir si la rose», p. 50)

Lorsqu'un énonciateur cite partiellement un discours autre, il peut, dans un souci de fidélité, marquer les «coupes» qu'il a opérées au moyen de points de suspension encadrés de parenthèses (mais non de tirets).

[34] Même détermination du côté de la CDU: «Il n’y aura pas de négociations sur le contenu, si le SPD n'accepte pas que notre candidate devienne chancelière (...). Le 
SPD doit reconnaître la réalité après les élections de Dresde» - élections qui ont augmenté l'avance de la CDU - a affirmé de son côté mardi 4 octobre le secrétaire général de la CDU, Volker Kauder, à la chaîne publique de télévision ARD.

(Le Monde, 04/Io/2005, consulté sur http://www.lemonde.fr/europe/article/2005/ Io/o4/les-negociations-dans-1-impasse_695784_32I4.html)

Il arrive également que la séquence marquée de nos signes soit le lieu du retour de la voix de l'énonciateur principal dans un discours direct. S'il s'agit d'attribution du dit, parenthèses et tirets se rencontrent de manière indifférente:

[35] Toubon a confirmé qu'il n'engagerait pas de réforme du code pénal pour protéger l'instruction. «Il n'est pas besoin de prévoir un nouveau texte - dit-il -, appliquons le code civil, il est efficace».

(Libération, 06/06/1995, p. I2; cité d'après Pétillon-Boucheron, 2003: 178)

[36] Certains candidats comme Brunot Mégret ou Gérard Freulet améliorent légèrement le score présidentiel de Le Pen. C'est «un mouvement en pleine expansion (assure-

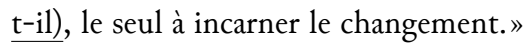

(Libération, I2/06/1995, p. 3; cité d'après Pétillon-Boucheron, 2003: 178)

En revanche, lorsqu'il s'agit de commenter le discours de l'autre ${ }^{8}$, seules les parenthèses ont été observées, qu'il s'agisse:

- d'expliciter certains éléments qui pourraient être opaques pour le lecteur:

[37] Alain Manoukian: «Il y a en tout cas une candidate que nous avons sélectionnée ici (lors de la première étape NDLR) qui est une graine de finaliste».

(Télé Moustique, $\mathrm{n}^{\circ}$ I3, 22-28/03/2003, p. 38)

- de donner un commentaire personnel sur le discours autre:

[38] LES PLUS: «Renaud a gardé malgré ses blessures le plaisir d'écrire.» (Est-ce vraiment un plus?)

(20 ans, $\mathrm{n}^{\circ} 193$, octobre 2002, p. 27)

- ou de donner des indications sur le comportement non verbal de l'énonciateur secondaire :

[39] Et moi, quand la caméra s'éloigne, je fais des grimaces et je mords les joues. (Rires.) (Public, $\mathrm{n}^{\circ} 8 \mathrm{O}, 2 \mathrm{I}-30 / 0 \mathrm{I} / 2005$, p. 94)

8. Les parenthèses au sein du discours direct peuvent être sources d'ambiguïté quant à l'attribution du dit; il n'est pas toujours évident de savoir si les séquences parenthésées appartiennent au discours cité ou au discours citant. Sur ce point, on se reportera utilement à Pétillon-Boucheron (2003: 259-266). 


\subsection{Renvois intra- et intertextuels}

Lorsque l'énonciateur désire renvoyer son lecteur à un autre endroit du texte, il le fait habituellement entre parenthèses:

[40] On ne sait toujours pas ce qu'est le vide, mais on commence à savoir ce qu'il fait: des expériences de plus en plus précises mesurent l'effet de ses fluctuations électromagnétiques (lire p. 50).

(Science et vie, $\mathrm{n}^{\circ}$ 1029, juin 2003, p. 49)

De même, les appels de note peuvent être encadrés de parenthèses:

[4I] [...] On a déjà vu pire - le pape en maillot à Castel Gondolfo par exemple $\left(^{*}\right)$.

$\left({ }^{*}\right)$ Voir le livre de photos «Le pape: dans l'intimité d'un homme» d'Adriano Bartoloni aux éditions Robert Laffont.

(Télé Moustique, $\mathrm{n}^{\circ} \mathrm{I3}, 22-28 / 03 / 2003$, p. 8)

Il en va de même lorsque l'auteur renvoie explicitement à un autre texte:

[42] Pas si grosse que cela, l'idée noire de notre lecteur (voir Le Moustique de la semaine dernière) d'envoyer le pape faire le bouclier humain à Bagdad [...].

(Télé Moustique, $\mathrm{n}^{\circ}{ }_{13}, 22-28 / 03 / 2003$, p. 7 )

[43] Lionel Florence, qui a écrit des textes pour Florent Pagny, Obispo, les Halliday (www.Lionelflorence.com).

(Télé Moustique, nº 13, 22-28/03/2003, p. 32)

Dans tous ces cas, nous n'avons relevé aucune occurrence de tirets.

1.4. Bilan

Une première constatation s'impose: le tiret ne semble pas avoir d'usages propres. Dans tous les cas où le tiret est possible, la parenthèse l'est aussi. Le contraire n'est cependant pas vrai. La parenthèse apparait, dans notre corpus, comme ayant des usages propres: les indications de naissance et de mort d'une personne, le commentaire sur un discours autre et les renvois intra- et intertextuels.

\section{Analyse syntaxique}

Notre analyse syntaxique abordera deux aspects du fonctionnement des éléments décrochés typographiquement. Nous verrons dans un premier temps leur fonction avant de revenir sur la question (classique) de leur caractère suppressible.

\subsection{Fonction des éléments décrochés}

Pour observer les rapports syntaxiques entre le segment linguistique marqué de nos signes et son environnement, nous nous baserons sur le modèle incidentiel développé par Wilmet (2010). 
On se rend compte que presque tous les usages de nos deux signes sont semblables. On y trouve:

- des appositions:

[44] La crise aidant, la dot - une tradition qui se maintient - devient hors de prix [...]. (La recherche. L'actualité des sciences, $\mathrm{n}^{\circ} 360$, janvier 2003, p. 23)

[45] Pour éviter les problèmes (enlèvement, harcèlement, etc.) les enfants de Michael Jackson ne sortent jamais sans petits masques en forme de papillon.

(Télé Moustique, $\mathrm{n}^{\circ}$ 13, 22-28/03/2003, p. 19)

- des déterminants caractérisants ${ }^{9}$ :

[46] De quoi s'agit-il? De l'histoire - mouvementée - d'un groupe de rock - imaginaire - qui répond au doux nom de «The Final Tap» (littéralement, "ponction lombaire»), filmée à la façon d'un documentaire.

(Télérama, $\mathrm{n}^{\circ}$ 2322, p. 71; cité d'après Pétillon-Boucheron, 2003: 163)

[47] Voyage au bout de la nuit traduit en hébreu et publié en Israël: l'antisémite qu'était Céline aurait certainement goûté le (petit) scandale suscité.

(Télérama, $\mathrm{n}^{\circ}$ 230I, p. 24; cité d'après Pétillon-Boucheron, 2003: 162)

- des compléments circonstanciels divers de l'énonciation:

[48] Bidji déclara que d'abord le banquier Raphäl - puisque c'était de lui qu'il s'agissait devrait s'engager à verser une grosse récompense.

(P. Grainville, Les forteresses noires, p. I45)

[49] Qu'ils soient conscients comme celui de Pro Flandria (sans parler de celui de Liberaal Appel, la dissidence ultra-droitière du VLD), ou malencontreux, les appels en direction de l'extrême-droite se répètent au nord du pays.

(Télé Moustique, $\mathrm{n}^{\circ}$ 13, 22-28/03/2003, p. 7)

- ou des compléments circonstanciels divers de l'énoncé:

[5०] C'est que la nature est finie, discrète - comme les bits de l'information.

(La recherche. L'actualité des sciences, $\mathrm{n}^{\circ} 360$, janvier 2003, p. 36)

[5I] «[...] Israël a retiré toutes ses forces du Liban sud (en mai 2000) conformément à la résolution 425 de l'ONU, ce que l'ONU a reconnu en juin 2000», a ajouté M. Pazner. (Le Monde, $14 / 03 / 2003$, consulté sur Internet, lien indisponible)

9. Terme utilisé par Wilmet (20I0) pour désigner l'ensemble des éléments qui se rapportent au nom et qui en réduisent l'extension en énoncé (ensemble des objets du monde auxquels un objet est applicable), par opposition aux déterminants quantifiants, qui se rapportent également au nom, mais qui en réduisent l'extensité (nombre d'objets du monde auxquels un objet est applicable). 
- des groupes coordonnés (sans que la fonction des éléments liés semble avoir une quelconque importance):

[52] Comme il n'y a que la peur du gendarme - et de l'amende - qui empêche certains de faire des bêtises, les sanctions sont renforcées.

$\left(\right.$ Maxi, $\mathrm{n}^{\circ} 898, \mathrm{I2}-\mathrm{I} 8 / \mathrm{oI} / 2004$, p. 34)

[53] En tout cas, ce que d'aucuns, en Irak, appellent la «résistance à l'occupation» prend de l'ampleur. Sans doute, les actes antiaméricains (et antibritanniques) plafonnentils désormais à douze/quinze par jour, ce qui n'est pas négligeable, mais surtout ils se font plus précis, plus sophistiqués et mieux ciblés.

(Le Soir en ligne, consulté sur http://archives.lesoir.be/irak-plusieurs-sortes-dhommes-ou-de-groupes-armes_t-20030819-ZoNF54.html)

[54] Ainsi, l'aspiran (ou riveirenc) du Languedoc, un vin peu coloré, peu tannique mais délicat, l'œillade (ou pacardan, ou araignan selon les lieux), le grenouillet du Cher, l'espinou d'Auvergne ne doivent leur salut qu’à quelques viticulteurs passionnés, ou à leur présence dans un décret d'appellation d'origine contrôlée (AOC).

(Science et vie, $\mathrm{n}^{\circ}$ I033, octobre 2003, p. II2)

- des groupes juxtaposés:

[55] Nous étions entrés de plain-pied dans un domaine interdit, et le monde du dehors, le monde lointain dont pour l'instant nous ne faisions pas partie, ne pourrait avoir pour nous que blâme et refus - n'avait pour nous que blâme et refus mais ne le savait pas: c'était notre tâche de le laisser dans l'ignorance.

(J. Harpman, La plage d'Ostende, p. 76)

[56] Prix? Vendredi $€_{\text {I2 }}$ en prévente ( $€_{\text {I5 sur place) }}$.

(Metro Summertime, no 609, oI-05/08/2003, p. I4)

On notera que la juxtaposition entre parenthèses ou tirets fonctionne différemment de la juxtaposition marquée de la simple virgule. Il semblerait qu'elle force une interprétation reformulative des groupes concernés, ce que confirment les exemples suivants :

[57] Ainsi, l'aspiran (ou riveirenc) du Languedoc, au vin peu coloré, peu tannique mais délicat, l'œillade (ou picardan, ou araignan selon les lieux), le grenouillet du Cher, l'espinou d'Auvergne ne doivent leur salut qu'à quelques viticulteurs passionnés, ou à leur présence dans un décret d'appellation d'origine contrôlée (AOC).

(Science et vie, $\mathrm{n}^{\circ}$ I033, octobre 2003, p. II2)

[58] La place où trône le roi avec son épouse est aussi bien celle de l'artiste et celle du spectateur : au fond du miroir pourraient apparaitre - devraient apparaitre - le visage anonyme du passant et celui de Velazquez.

(M. Foucault, cité d'après Pétillon-Boucheron, 2003: 169) 
Ceci permet notamment la «quantification a posteriori»:

[59] Il y a cette loi française, votée il y a quelques années, qui propose, pour calmer les chiens pitbulls agressifs (la plupart), de les castrer.

(Télé Moustique, $\mathrm{n}^{\circ} \mathrm{13}, 22-28 / 03 / 2003$, p. 14)

Dans cet exemple, la plupart indique après coup que les pitbulls sont pour la plupart agressifs. Dans ce cas, la plupart fonctionne comme un pronom placé après un syntagme dont il est homoréférentiel ${ }^{10}$.

- et enfin, des phrases incidentes ${ }^{11}$ :

[6o] Et puis, je préfère donner que recevoir - j’aime bien voir le plaisir que cela fait, un peu comme en amour...

(Télé Moustique, $\mathrm{n}^{\circ}$ 2, I0-16/or/2004, p. 5)

[6I] Car s'il s'en trouve (les rues des capitales en sont pleines le week-end) pour souligner que George W. Bush n'est pas un bon président des États-Unis, il en est pour rappeler que George W. Bush n'est pas le bon président des États-Unis.

(Télé Moustique, $\mathrm{n}^{\circ}{ }_{13}, 22-28 / 03 / 2003$, p. 5 )

Il est cependant des contextes qui ne semblent permettre que les parenthèses. C'est notamment le cas:

- des points d'interrogation, d'exclamation ou de suspension utilisés seuls:

[62] Le gagnant signe un contrat «platinium» qui lui assure notamment une meilleure promotion (!).

(Télé Moustique, $\mathrm{n}^{\circ}$ 13, 22-28/03/2003, p. 32)

[63] Même détermination du côté de la CDU: «Il n’y aura pas de négociations sur le contenu, si le SPD n'accepte pas que notre candidate devienne chancelière (...). Le SPD doit reconnaitre la réalité après les élections de Dresde» - élections qui ont augmenté l'avance de la CDU - a affirmé de son côté mardi 4 octobre le secrétaire général de la CDU, Volker Kauder, à la chaîne publique de télévision ARD.

(Le Monde, 04/10/2005, consulté sur http://www.lemonde.fr/europe/article/2005/ Io/o4/les-negociations-dans-1-impasse_695784_3214.html)

10. On pourrait également penser que le groupe entre parenthèses est ici en prédication seconde sur les chiens pitbulls agressifs. Il est vrai qu'en contexte de reformulation la limite entre maillage et prédication est ténue, comme, par exemple, dans: «Le chardonnay, le melon (muscadet), le gamay et une dizaine d'autres cépages [...]» (Science et vie, n I033, octobre 2003, p. IIO). Voir François (2006: I03-I06).

11. Nous appelons, à la suite de Wilmet (2010: 672-679), phrase incidente toute phrase insérée dans une autre sans y occuper une quelconque fonction syntaxique. Elle se distingue de la phrase incise qui, elle, est complément de l'énonciation; le cas prototypique en est l'attribution du dit dans le discours rapporté (dit-il, etc.). 
- des segments inférieurs aux mots:

- lettres:

[64] Le résultat est (d)éton(n)ant et on se demande ce que la version peroxydée peut bien nous réserver.

(Télé Moustique, nº 8, 24-28/02/2006, p. 32)

- morphèmes:

[65] L'été est la saison privilégiée pour partir à la (re)découverte du patrimoine touristique de la Belgique.

(Metro Summertime, $\mathrm{n}^{\circ}$ 609, oI-05/08/2003, p. 16)

- morphèmes ponctués:

[66] Mon merveilleux poisson doré: quels beaux jours que tous les jours depuis que nous nous sommes (re?)trouvés!

(Libération, cahier de la Saint-Valentin, I4/02/1995; cité d'après Pétillon-Boucheron, 2003: 157)

- de la ponctuation de $\operatorname{mot}^{12}$ :

[67] Zorra $\mathrm{m}\left({ }^{\prime}\right)$ a muse.

(Elle, 20/o8/2004, p. I4)

- ou des phrases typographiquement marquées:

[68] M.C.: Êtes-vous heureuse aujourd'hui?

C.: Non... Enfin, je dis peut-être ça parce que je suis très fatiguée. (Rires.)

(Marie-Claire, $\mathrm{n}^{\circ}$ 618, février 2004, p. 42)

[69] Tout ça m'a inspiré un film. (Il raconte le film interminablement, au cas où on ne l'aurait pas compris.)

(20 ans, $\mathrm{n}^{\circ} 193$, octobre 2002, p. 9)

Par ailleurs, les parenthèses semblent la seule solution pour les noms ou syntagmes nominaux déterminants caractérisants sans préposition:

[70] Sur le terrain, deux obus de mortier ont été tirés dimanche matin contre une colonie du sud de la bande de Gaza, et deux Palestiniens recherchés ont été arrêtés par l'armée israélienne près de Bethléem (Cisjordanie).

(Le Monde, 14/03/2003, consulté sur Internet, lien indisponible)

12. Nous appelons ponctuation de mot l'ensemble des signes graphiques marquant l'organisation du mot graphique (que ce soit pour en marquer les frontières ou pour signaler la composition morphologique); on y retrouve l'espace, l'apostrophe et le trait d'union. Pour une discussion plus fournie, voir François (2006: 53-57). 
[71] La députée Karin Lalieux (PS) a présenté une proposition de loi obligeant les banques à fournir gratuitement et sans conditions un service bancaire de base.

(Télé Moustique, $\mathrm{n}^{\circ}$ 13, 22-28/03/20 03, p. 14)

Certains adverbes enfin semblent réservés aux parenthèses:

[72] À vrai dire, il ne manque que la dédicace sur ce cliché ${ }^{13}$ en noir et blanc que l'on verrait volontiers encadré sur le buffet de sa maman ou accroché à l'entrée d'un vieux cinéma aux fauteuils de velours pourpre (déjà!).

(Télé Moustique, $\mathrm{n}^{\circ}{ }_{13}, 22-28 / 03 / 2003$, p. 8)

Dans cet exemple, déjà se rapporte sémantiquement à pourpre (en allusion à la pourpre cardinalice). En aucun cas, la séquence n'est équivalente à de velours déjà pourpre, qui signifierait que le velours était à ce moment, et est encore, pourpre. Dans ce dernier contexte, nous dirions à la suite de Wilmet (2010: 349-350 et 5OI-502) que l'adverbe est incident à la relation qui unit le déterminant (pourpre) à son noyau (velours). Or ici, l'adverbe ne se rapporte sémantiquement qu'au seul mot pourpre à l'exclusion de tout autre. Son incidence est ici problématique, de même que sa position ${ }^{14}$.

Notre corpus semble assez clairement montrer que le fonctionnement syntaxique des deux signes est différent. La parenthèse peut marquer tous les éléments de la chaîne écrite, de la simple lettre à plusieurs phrases, alors que le tiret ne marque que des segments supérieurs ou égaux au mot (graphique) et inférieurs ou égaux à la phrase. Par ailleurs, la parenthèse semble être la seule à pouvoir encadrer des éléments syntaxiquement problématiques comme notre déjà.

\subsection{Caractère suppressible des éléments décrochés}

De nombreux traités de ponctuation estiment que l'élément placé entre parenthèses ou tirets est suppressible. Pétillon-Boucheron (2003: I8I) fait de ce critère une condition «nécessaire mais non suffisante» pour qu'un élément soit décrochable.

Serça (20I0: 72) montre cependant que certains éléments décrochés sont, chez Proust, essentiels à la compréhension de la phrase d'accueil.

[73] Et pourtant voici qu'une légère contrariété ou un malaise physique - en l'incitant à considérer le moment présent comme un moment exceptionnel, en dehors de la règle, où la sagesse même admettrait d'accueillir l'apaisement qu'apporte un plaisir et de donner congé, jusqu'à la reprise utile de l'effort, à la volonté - suspendait l'action de celle-ci qui cessait d'exercer sa compression [...].

(M. Proust, cité d'après Serça, 20IO : 72)

13. L'article parle d'une photo de Jean-Paul II alors qu'il était acteur en Pologne.

14. La seule solution qui tienne dans notre modèle est de considérer qu'il s'agit d'une phrase incidente incomplète. 
15. Tous les exemples de ce type que nous avons relevés contiennent des parenthèses et non des tirets. Ils sont cependant trop peu nombreux pour que nous puissions en tirer une quelconque conclusion.

16. Le seul souci de grammaticalité se situe au niveau des accords. Voir Pétillon-Boucheron (2003: 217-222).

Il semble donc que les éléments encadrés par des parenthèses ou des tirets sont (syntaxiquement) suppressibles quel que soit le signe utilisé. Serça (2010: I03) affirme cependant que «si les deux signes encadrent un élément suppressible, celui

Dans l'exemple cité, celle-ci renvoie à la volonté, qui se trouve à l'intérieur de l'élément décroché. Privée de la séquence placée entre tirets, la phrase deviendrait incompréhensible par l'absence de l'élément qui permet la résolution de l'anaphore.

Cependant, si l'énoncé est incompréhensible, il n'en est pas moins grammatical. Sa structure syntaxique est correcte. Ce n'est que textuellement qu'il est incohérent. Toute la question est donc celle de la définition de la suppressibilité.

La grammaire scolaire nous a habitués à une définition extrêmement restrictive de ce critère syntaxique. La plupart de nos exemples montrent des segments décrochés clairement suppressibles. Une série d'autres mettent en revanche au jour des éléments réputés essentiels: les lettres (exemple [64]), les morphèmes (exemples [65] et [66]), les ponctuations de mot (exemple [67]). D'autres exemples corroborent cette constatation ${ }^{15}$.

[74] C'est un texte que l'eau ramifie, l'eau et le désir qui s'écoule en lui. C'est un mouvement (de) vague; et si c'est cela, alors il ne s'agit pas des rouleaux de la pleine mer mais plutôt des larmes ultimes sur les galets.

(P. Grouix, mémoire de DEA-Lettres, cité d'après Pétillon-Boucheron, 2003: I66)

[75] La sexion est (la) sexualité.

(A. Green, cité d'après Pétillon-Boucheron, 2003: 162)

En [74], nous trouvons une préposition entre parenthèses et en [75] un déterminant quantifiant.

Si on regarde cependant les choses de plus près, on se rend compte que, privés des éléments décrochés, les phrases envisagées sont toujours acceptables. Les éléments inférieurs au mot nous invitent à une double lecture du texte en proposant une alternance syntagmatique de deux termes graphiquement proches. En [74], le décrochement de de n'est possible que parce que vague peut être soit un adjectif (utilisé sans préposition), soit un nom (employé avec préposition). En [75], la mise entre parenthèses de l'article n'est envisageable que parce que la construction attributive permet soit une construction avec un déterminant $\varnothing$, soit avec la.

Ainsi, chacun des éléments encadrés de nos signes est suppressible, au sens que lui donne Le Goffic (I993), dans la mesure où l'énoncé, privé de ces éléments, reste grammatical $^{16}$. 
[= l'élément suppressible] qui est isolé par les tirets n'est pas destiné à être enlevé». Selon elle, «[1]e terme fait partie de la phrase d'accueil qui perdrait indéniablement de son sens avec sa suppression». Le problème est que toute suppression d'un quelconque élément linguistique entraîne une perte de sens. Quant au caractère indéniable de celle-ci, il semble éminemment subjectif. Catach (1996: 74) défend même l'idée que les parenthèses véhiculent l'élément «le plus expressif du message». Supprimer leur contenu créerait donc une indéniable perte de sens. Par ailleurs, dans la plupart de nos exemples, la perte sémantique qu'entraînerait l'effacement de l'élément décroché ne semble pas dépendante du signe utilisé. Comparons les exemples [52], [53], [60] et [6r] aux exemples [52a], [53a], [6oa] et [6ra] ci-dessous:

[52a] Comme il n'y a que la peur du gendarme qui empêche certains de faire des bêtises, les sanctions sont renforcées.

(Maxi, $\mathrm{n}^{\circ} 898, \mathrm{I} 2-\mathrm{I} 8 / \mathrm{oI} / 2004$, p. 34)

[53a] En tout cas, ce que d'aucuns, en Irak, appellent la «résistance à l'occupation» prend de l'ampleur. Sans doute, les actes antiaméricains plafonnent-ils désormais à douze/quinze par jour, ce qui n'est pas négligeable, mais surtout ils se font plus précis, plus sophistiqués et mieux ciblés.

(Le Soir en ligne, consulté sur http://archives.lesoir.be/irak-plusieurs-sortes-dhommes-ou-de-groupes-armes_t-20030819-ZoNF54.html)

[6oa] Et puis, je préfère donner que recevoir...

(Télé Moustique, $\mathrm{n}^{\circ}$ 2, 10-16/or/2004, p. 5)

[6ra] Car s'il s'en trouve pour souligner que George W. Bush n'est pas un bon président des États-Unis, il en est pour rappeler que George W. Bush n'est pas le bon président des États-Unis.

(Télé Moustique, $\mathrm{n}^{\circ}{ }_{13}, 22-28 / 03 / 2003$, p. 5 )

Il semble au vu de ces exemples que, pour des usages sémantiques équivalents, la perte de sens est équivalente.

Serça (2010: 103) ajoute d'ailleurs: "Cette nuance entre parenthèses et tiret double n'indique qu'une tendance en langue, sans préjuger d'usages divers, voire contradictoires en discours.» Dans notre cadre théorique, le système en langue implique nécessairement des effets congruents en discours. Un effet de discours contradictoire impliquerait nécessairement une remise en question de la modélisation en langue qui a été proposée. Le critère ne peut donc, à notre sens, être retenu pour distinguer nos deux signes ${ }^{17}$.

17. Le seul argument proprement linguistique que nous pourrions retenir serait que les anaphores renvoyant à un élément de la séquence décrochée (comme dans l'exemple [73]) ne sont possibles qu'avec des tirets. Ce type de configuration semble extrêmement rare. Ni notre corpus, ni celui de Pétillon-Boucheron (2003) n'en donne d'occurrence. Face à cette carence de données, rien ne permet a priori d'exclure ce type d'usage avec les parenthèses (même si intuitivement cela semble moins acceptable). 


\section{Fonctionnement typographique}

La ponctuation interne des séquences entre parenthèses ou tirets est celle qui serait attendue dans des séquences non marquées de ces signes. On retrouve ainsi des virgules séparant des éléments coordonnés (exemple [76]) ou encadrant des circonstanciels (exemple [77]).

[76] La configuration actuelle - une bande sortante, une bande entrante et une bande qui change d'affectation entre le matin et le soir - changera-t-elle au cours des travaux?

(Télé Moustique, $\mathrm{n}^{\circ}$ 53, 2003, p. 4)

[77] La dernière fois où Svan Nielsen m'a parlé au téléphone (nous nous comprenions avec difficulté car, par mutuelle coquetterie, nous nous entêtions à emprunter chacun la langue de l'autre. Or son français est Fleuve noir et mon danois date de Kierkegaard), il m'a dit: «Vous aurez de mes nouvelles, Monsieur Franck».

(B. Franck, cité d'après Pétillon-Boucheron, 2003: 187)

De même, si une citation se trouve dans le segment concerné, les guillemets la marquent normalement :

[78] À 20 ans, après deux années plutôt lamentables à l'université, il s'engage pour le Vietnam et trouve sa voie - une trajectoire comparable à celle de Collin Powell, le général en retraite qui dirige la diplomatie américaine. Comme lui, il est blessé et médaillé dans l'infanterie, ce qui explique son enthousiasme pour la «doctrine Powell»- force prépondérante, mission clairement définie.

(Le Soir, 17/03/2003, p. 2)

[79] «Si t'es pas du coin, touche pas à mon quotidien.» C'est la new campagne publicitaire du groupe Sud Presse. Ça a l'air rigolo et sympa, mais hélas, ça ne l'est pas. C'est, qu'on le veuille ou non, raciste. Au départ d'un vieux slogan antiraciste («Touche pas à mon pote»), mais parfaitement raciste.

(Télé Moustique, $\mathrm{n}^{\circ}$ 13, 22-28/03/2003, p. 14)

Le tiret parenthétique initial n'est jamais suivi ni précédé d'aucune ponctuation. La parenthèse ouvrante peut au contraire être précédée d'un point et suivie d'une majuscule si la séquence encadrée est une phrase graphique:

[80] Tout ça m'a inspiré un film. (Il raconte le film interminablement, au cas où on ne l'aurait pas compris.)

(20 ans, $\mathrm{n}^{\circ} 193$, octobre 2002 p. 9)

L'exemple ci-dessus illustre en outre la présence possible d'un point marquant la finale de phrase devant une parenthèse fermante, usage interdit au tiret. Dans ce cas, la parenthèse fermante marque la fin du paragraphe (comme ci-dessus) ou est suivie d'une majuscule: 
[8I] Attendez: je n'ai jamais dit non à 20 millions de dollars! (Il rit bruyamment.) Je ne complique pas la vie, mais j'ai tendance à faire ce que je veux.

(20 ans, $\mathrm{n}^{\circ}$ 193, octobre 2002, p. 10)

Nos deux signes en position finale peuvent par ailleurs être précédés:

- de points d'exclamation:

[82] Nous marchions depuis une heure déjà, et ces quelques lignes - oh! si peu! - sont ce que je peux reproduire d'une conversation longue et rêveuse.

(J. Harpman, La plage d'Ostende, p. 272)

[83] La carte des vins (35 pages!) couvre les meilleurs vignobles français, avec un détour par le bout du monde.

(Gaël, n ${ }^{\circ}$ I83-I, janvier 2004, p. I5)

- de points d'interrogation:

[84] Dans ce collège - mais peut-être en était-il ainsi ailleurs? -, on apprenait que l'acte d'amour trouvait sa fin ultime dans la reproduction.

(J.-L. Outers, L'ordre du jour, p. 179)

[85] Contrairement à Wolfram et Fredkin, qui depuis de nombreuses années n'ont rien publié dans un journal scientifique à comité de lecture, Lloyd joue (encore?) cette carte à fond.

(La recherche. L'actualité des sciences, $\mathrm{n}^{\circ} 360$, janvier 2003, p. 37)

- et de points de suspension:

[86] Chez les personnes âgées, c'est même trois ou quatre verres par jour (pas plus...) qui retarderaient l'apparition du gâtisme ou d'une maladie d'Alzheimer.

(Science et vie, $\mathrm{n}^{\circ} \mathrm{IO} 33$, octobre 2003, p. II4)

On notera que ces signes ne marquent pas forcément une finale de phrase, au contraire du point. Ils peuvent bien sûr le faire et ce sont les cas les plus courants:

[87] Putain de Vercors! On va péter le pare-brise si ça continue! L'essuie-glace gauche a morflé. Tu vois le croisement? Fais gaffe, là, devant, devant... Devant! Ça vient vers nous! Freiiiiine! Jusqu'au choc. Mou, le choc. Lourd et mou.

(D. Pennac, Monsieur Malaussène, p. 402)

Mais ils peuvent également être équivalents à la virgule (ils marquent alors l'interrogation, l'exclamation ou la suspension en plus de segmenter le texte):

- pour séparer le discours cité de l'incise dans les discours directs:

[88] Non, non... légèrement acidulé, juste ce qu'il faut, exquis, exquis! répondit Chandor. (P. Grainville, Les forteresses noires, p. 244) 
- pour séparer des phrases énumérées:

[89] Là pourtant surgissant de cette brume, la brusque violence de la terreur, de l'horreur... je hurle, je me débats... qu'est-il arrivé? que m’arrive-t-il?

(N. Sarraute, Enfance, p. 25)

- pour séparer des groupes juxtaposés ${ }^{18}$ :

[90] Quoi faire d'un tel trésor? Le moindre achat un peu tapageur provoquerait la suspicion des parents. D'où viennent cette somptueuse Mobylette? ce magnétophone? cette chaîne stéréo?... Au moindre indice, ils étaient brûlés.

(P. Grainville, Les forteresses noires, p. 159)

Ils peuvent également marquer des éléments internes à la phrase:

[9I] C'était si saugrenu d'être là à guetter quoi? tout seul.

(P. Grainville, Les forteresses noires, p. 272)

[92] Je fus surprise par l'indocilité de mon cœur qui ne cessait pas de battre. Iseult, pourtant! glisse morte à côté de Tristan?

(J. Harpman, La plage d'Ostende, p. 289)

[93] Jeune surdoué ayant lâché l'université, il intégra l'US Air Force comme pilote de chasse. Passé consultant en informatique, il créa son entreprise, devint millionnaire et... fut nommé professeur d'informatique au Massachussets Institut of Technologie (MIT), où il dirigea le laboratoire de science informatique.

(La recherche. L'actualité des sciences, $\mathrm{n}^{\circ}$ 360, janvier 2003, p. 35)

Nous voyons donc que les points d'interrogation, d'exclamation et de suspension ne marquent pas forcément les finales de phrase ${ }^{19}$.

Dans les exemples [82] à [86], nous pouvons constater que le signe fermant est toujours suivi d'une minuscule. Par ailleurs, la séquence encadrée de nos signes ne commence pas par une majuscule. On peut donc affirmer sans trop de risque que, dans ce cas, nos points ne marquent pas une finale de phrase, mais sont des signes internes à celle-ci.

On peut trouver ces points comme marques de finale de phrase, mais à l'instar $\mathrm{du}$ point final, cet usage semble être réservé aux parenthèses ${ }^{20}$ :

[94] LES PLUS: «Renaud a gardé malgré ses blessures le plaisir d'écrire.» (Est-ce vraiment un plus?)

(20 ans, $\mathrm{n}^{\circ}$ 193, octobre 2002, p. 27)

18. L'accord du verbe au pluriel force à interpréter cet énoncé comme ayant plusieurs sujets.

19. Voir François (2006: 132-166).

20. Dans les deux exemples cités, la parenthèse est le dernier signe du paragraphe. 
[95] Vous n'avez plus goût à la vie. Ce n'est pas qu'il soit irremplaçable, mais voilà une bonne occasion d'en finir... (Attention, c'est pour rigoler, n'allez pas vous suicider pour un bidon!)

(20 ans, $\mathrm{n}^{\circ} 193$, octobre 2002, p. 75 )

La ponctuation suivant la parenthèse fermante dépend du contexte d'apparition de la séquence parenthésée. Ainsi, dans les cas de parenthèses de mot, si l'élément morphologique n'est pas séparé d'un blanc, la parenthèse ne connaîtra pas ce signe non plus, tandis que si une espace ${ }^{21}$ est nécessaire, celle-ci apparaîtra aussi entre la parenthèse et les éléments de composition morphologique. Il se produira la même chose au sein de la phrase. Si une espace est attendue après la séquence, une espace apparaitra après la parenthèse:

[96] L'acquisition de la nationalité belge peut également être obtenue par mariage avec un conjoint belge. Dans ce cas il faut avoir résidé avec lui (!) en Belgique pendant trois années au minimum ou pendant au moins six mois mais, dans cette dernière hypothèse, à la condition expresse d'avoir, au préalable, été autorisé, depuis au moins trois ans, à séjourner en Belgique plus de trois mois (bravo à ceux qui ont suivi).

(Metro Summertime, no 609, 0I-05/08/2003, p. II)

En revanche, si un autre signe est attendu, c'est celui-là qui apparâtra: virgule (exemple [97]), point (exemple [96] ci-dessus)...

[97] «Si t'es pas du coin, touche pas à mon quotidien.» C'est la new campagne publicitaire du groupe Sud Presse. Ça a l'air rigolo et sympa, mais hélas, ça ne l'est pas. C’est, qu'on le veuille ou non, raciste. Au départ d'un vieux slogan antiraciste («Touche pas à mon pote»), mais parfaitement raciste.

(Télé Moustique, $\mathrm{n}^{\circ}$ 13, 22-28/03/2003, p. 14)

Le tiret final fonctionne sur le même principe, si ce n'est qu'il ne peut marquer des séquences inférieures au mot graphique (voir ci-dessus) et qu'il n'est pas réalisé devant un point final ou des points d'interrogation, d'exclamation ou de suspension suivis d'une majuscule (voir ci-dessous). On retrouve donc après lui une espace ou une virgule.

[98] Cet effet reste néanmoins très mal cerné, à la fois sur le plan des concentrations - qui varient du simple au double, voire plus - et sur celui des mécanismes impliqués.

(La recherche. L'actualité des sciences, $\mathrm{n}^{\circ}$ 360, janvier 2003, p. 15 )

[99] Quand il entreprit sa brève toilette - se laver les dégouttait -, Raphaël avait probablement perçu, brouillé par le ruissellement des robinets, ce rythme infime, ce léger murmure de musique... le phénomène s'était peut-être produit déjà une ou deux fois. (P. Grainville, Les forteresses noires, p. IO-II)

21. Nous considérons l'espace (mot féminin dans le jargon typographique) comme un signe de ponctuation de mot marquant les limites entre ceux-ci en l'absence d'autre ponctuant. Voir François (2006) ou, pour une argumentation plus fouillée, Anis (1988), Védénina (1989), Catach (1996) ou Dahlet (2003). 
Par ailleurs, les parenthèses sont un signe double constitué d'un signe ouvrant «(» et d'un signe fermant «)». Toute parenthèse ouverte est normalement refermée (voir tous nos exemples de parenthèses!).

Le tiret, en revanche, ne connaît qu'une seule forme «-», aussi bien pour le signe ouvrant que pour le signe fermant:

[Ioo] J'en ai fait un [duo] avec Cliff Richard - Danny Boy - que j'interprète sur scène avec lui qui chante sur grand écran!

(Télé Moustique, $\mathrm{n}^{\circ}$ 2, I0-16/or/2004, p. 5)

Mais il arrive également que seul le tiret ouvrant soit réalisé. C'est le cas lorsque la séquence introduite par un tiret se trouve en fin de phrase graphique (exemples [IoI] et [IO2]) ou en fin de séquence parenthésée (exemple [IO3]).

[IoI] Autre objet significatif, la hache en cuivre (à droite) d'Ötzi - la plus ancienne hache préhistorique retrouvée en Europe avec manche et fixations intacts.

(National Geographic France, n ${ }^{\circ}$, février 2003)

[IO2] Et puis, je préfère donner que recevoir - j’aime bien voir le plaisir que cela fait, un peu comme en amour...

(Télé Moustique, nº 2, 10-16/or/2004, p. 5)

[103] Parce que ce sont eux qui prennent le plus d'immunosuppresseurs (des médicaments destinés à contrôler le rejet - NDLR)

(Télé Moustique, $\mathrm{n}^{\circ}$ 13, 22-28/03/2003, p. 38)

C'est ce fonctionnement typographique particulier qui nous fait préférer, à la suite de Pétillon-Boucheron (2003), l'appellation de «tiret parenthétique» à celle, plus traditionnelle, de «tiret double» ${ }^{22}$.

Nous voyons donc qu'au niveau de la combinaison avec d'autres signes de ponctuation, les parenthèses peuvent encadrer des phrases graphiques entières alors que cet usage est interdit aux tirets qui ne peuvent marquer que des séquences internes à la phrase. Par ailleurs, toute parenthèse ouverte est refermée alors que les séquences marquées du tiret n'ont pas de signe spécifique fermant si leur finale correspond à la limite de la phrase.

22. Selon une optique traditionnelle (voir notamment Halkin, I948), il s'agirait d'une règle typographique. Certes, on la retrouve dans les traités de typographie (Imprimerie nationale, 2002, par exemple), mais cela ne suffit pas, à notre sens, à expliquer pourquoi le tiret s'efface devant le point et non la parenthèse. De plus, certains auteurs (Anis, I988; Doppagne, I998; Védénina, 1989; entre autres) donnent de cette disparition du tiret fermant une explication proprement linguistique : l'«absorption» (Tournier, I980) de ce dernier par les ponctuations fortes. Dans les deux cas, le tiret conserve l'appellation de «tiret double», nous y reviendrons (partie 4 ). 


\section{Explication des différences}

Il semble évident que parenthèses et tirets marquent le même type de phénomène, ce décrochement énonciatif dont parle Pétillon-Boucheron. La preuve en est que tout tiret peut être remplacé par une parenthèse.

Cependant, nous l'avons vu, l'inverse n'est pas vrai. Comment expliquer cela? Pour répondre à cette question, résumons les différences que nous avons observées :

- au niveau pragmatique et sémantique, certains usages sont réservés aux parenthèses: les indications de renvoi à l'intérieur et à l'extérieur du texte, les signes de ponctuation utilisés seuls, les commentaires sur le discours d'autrui et les dates de naissance et de mort;

- au niveau syntaxique, nous avons vu que le tiret marque toujours des segments supérieurs au mot et inférieurs à la phrase intégrés syntaxiquement, alors que la parenthèse marque en plus des caractères, des morphèmes et des phrases entières;

- au niveau du fonctionnement typographique, nos constatations syntaxiques ont été renforcées: la parenthèse est la seule à accueillir des phrases graphiques entières; par ailleurs, les parenthèses vont toujours par paire - et distinguent le signe ouvrant du signe fermant -, alors que la séquence ouverte par un tiret n'est pas refermée par le même signe si celui-ci coïncide avec une limite de phrase (il ne connaît d'ailleurs qu'une seule forme).

Pour expliquer les différences de niveau de marquage, Tournier (I980) parle de «loi d'absorption»:

[...] il existe des signes qui ne peuvent apparaître l'un à côté de l'autre, bien que comportant des ponctuants [signifiants] et des ponctuances [signifiés] différents : dans de tels cas, un seul ponctuant est réalisé et il se charge alors de sa ponctuance propre et des autres.

Et il cite l'exemple de la virgule isolant un complément circonstanciel placé en fin de phrase, pour laquelle la virgule de clôture du segment est «absorbée» par le point. Dahlet (2003: 3I-32) invoque dans le même sens le "processus de résorption»:

Un signe est résorbé par un autre, morphologiquement différent, et en général de portée supérieure. La condition étant la contiguïté du point d'occurrence sur la chaîne écrite. C'est le cas des segments détachés à l'avant [...] ou à l'arrière $[. .$.$] , de sorte que le$ signe résorbé peut se trouver aussi bien sur la borne de gauche que sur celle de droite ${ }^{23}$.

23. Ce phénomène est parfois expliqué par une règle typographique (voir le point 4 ci-dessus). Il nous semble que cette seule explication ne saurait suffire à expliquer la régularité absolue de l'emploi. D'autres règles, purement typographiques, telles que l'impossibilité de placer une virgule devant des points de suspension ou de placer la ponctuation forte après les guillemets fermants (lorsque le segment cité est lui-même constitué d'une ou plusieurs phrases graphiques) connaissent en effet de nombreuses exceptions (voir François, 2006). 
Ce type d'explication permet assez bien de comprendre pourquoi le tiret final n'est pas réalisé dans certains cas, mais cela n'explique pas d'où provient la différence de fonctionnement.

Serça (2010: I03) nous propose une autre piste intéressante. Selon elle, le tiret double serait «du côté de l'extraction d'éléments homogènes et les parenthèses du côté de l'insertion d'éléments hétérogènes». En effet, le tiret semble se spécialiser dans le marquage d'éléments appartenant à la structure canonique de la phrase et les parenthèses sont le seul signe à pouvoir indiquer un changement de voix. Le problème est que la parenthèse peut aussi marquer des éléments syntaxiquement intégrés et n'indique pas forcément la polyphonie. Le tiret semble donc bien du côté de l'homogénéité, mais les parenthèses relèvent aussi bien de celle-ci que de l'hétérogénéité.

\subsection{Une différence de système...}

La solution se trouve à notre avis du côté du système de la ponctuation.

Afin de mieux entendre de quoi il retourne, il nous semble important de faire un petit détour du côté de la définition de celle-ci. Nous avons défini la ponctuation (François, 2006: 50) comme l'ensemble des signes typographiques servant à organiser physiquement le texte. Cette définition est large et englobe tous les niveaux de marquage physique, du mot au texte. À partir de cette définition, il nous a semblé que le plus évident était d'organiser le système en fonction du niveau marqué par le signe considéré ${ }^{24}$. À un premier niveau, nous trouvons la ponctuation de mot qui organise les unités inférieures ou égales au mot - il s'agit de l'espace, l'apostrophe et le trait d'union. Ensuite, nous avons la ponctuation de phrase qui marque les segments linguistiques strictement supérieurs au mot et inférieurs ou égaux à la phrase - la virgule, le point-virgule, le couple majuscule et point, les points d'interrogation, d'exclamation et de suspension ainsi que les deux-points ${ }^{25}$. Enfin, la ponctuation de texte marque les séquences strictement supérieures à la phrase - toutes les marques de mise en paragraphes, en chapitres, etc.

D'après ce que nous avons observé, il apparaît que le tiret parenthétique est assez clairement un signe de phrase, puisqu'il marque des séquences supérieures au mot et inférieures à la phrase ${ }^{26}$. En revanche, la parenthèse semble «se balader» entre les niveaux et n'appartenir à aucune des catégories que nous avons définies. En tant qu'elle marque un phénomène énonciatif, nous l'appellerons signe de l'énonciation, i. e. signe qui se balade entre les niveaux de marquage syntaxique et marque un phénomène énonciatif (les guillemets, marques par excellence de l'hétérogénéité discursive, ont aussi cette faculté de marquer des éléments très

24. C’est ce que Mourad (I999) appelle le pouvoir de segmentation physique.

25. Tous ces signes n'ont pas le même statut, voir ci-dessous.

26. Ceci explique, à notre sens, l'impression de Serça (2010: I03), pour qui le terme marqué du tiret «fait partie de la phrase d'accueil» et est «du côté de l'extraction d'éléments homogènes». 
divers dans le texte ; il est donc assez normal de leur faire rejoindre les parenthèses dans l'ensemble tel que nous venons de le définir).

\section{2. ...qui explique les fonctionnements typographiques différents...}

C'est cette différence de niveau de marquage qui explique, selon nous, les variations de fonctionnement entre les deux signes.

En tant que signes de l'énonciation, les parenthèses peuvent marquer tous les niveaux d'organisation linguistique y compris les segments intraphrastiques. C'est pourquoi elles peuvent toujours se substituer aux tirets. En revanche, le tiret, limité dans sa portée, ne peut commuter avec les parenthèses que si elles encadrent des segments internes à la phrase.

Par ailleurs, comme il s'agit d'un signe baladeur, rien ne permet de prévoir la limite de la portée d'une parenthèse ouverte, d'où la nécessité de refermer les séquences parenthésées.

En revanche, le tiret fonctionne comme un signe de phrase. Pour bien comprendre cela, il importe de se pencher sur la structure interne de cet ensemble. D'abord, nous avons un premier système composé de la virgule, du point-virgule et du couple majuscule point, marquant trois niveaux hiérarchisés d'interruptions graphiques ${ }^{27}$. Le deuxième ensemble est composé des signes qui marquent les mêmes niveaux, mais donnent en plus une information sémantique ou énonciative; on y retrouve les points d'interrogation, d'exclamation et de suspension, les deux-points ${ }^{28}$ et le tiret parenthétique.

Dans le système tel qu'il fonctionne en français, toute séquence ouverte au moyen d'un de ces signes se termine soit lorsque ce même signe est répété, soit lorsqu'un signe de niveau supérieur clôt une séquence plus importante. On peut observer ce phénomène avec la virgule marquant l'incise par exemple:

[104] Mais les troupes, dit-on, ont le moral et aussi sont impatientes de passer à l'action. (Le Soir, $17 / 03 / 2003$, p. 7)

[ios] - Ils sont jeunes, dit-il sobrement. Et libres. (J. Harpman, La plage d'Ostende, p. Ioo)

Dans le premier cas, l'incise commence par une virgule et se termine par le même signe alors que la citation se poursuit. Dans le second cas, si la virgule marque toujours la limite initiale, le point clôt en même temps la phrase citée et l'incise.

27. Nous préférons ce terme d'interruption graphique à celui de pause graphique que nous utilisions précédemment (François, 2006), en ce qu'il montre clairement que ce marquage n'entretient aucun lien direct avec les pauses orales.

28. Bien que la grammaire normative interdise aux deux-points de porter plus loin à droite que le point final, cet usage n'est pas rare. Nous réservons donc à ce signe un traitement particulier de "signe à débordement» (voir François, 2006: 166-I88). 
Le tiret fonctionne de la même manière. Lorsque la fin du segment marqué du tiret coïncide avec la fin de la séquence supérieure - la phrase -, le point marque, en même temps que le terminus ad quem de la phrase, la limite terminale de la séquence introduite par le tiret.

\section{3. ...et les différences d'usage}

La différence en termes de système explique d'abord les divergences de fonctionnements syntaxique et typographique, mais cet écart explique aussi sans doute les usages sémantiques distincts de nos deux signes. En effet, en tant que signe de l'énonciation, la parenthèse est apte à encadrer n'importe quelle séquence, alors que le tiret impose l'intégration syntaxique de celle-ci dans la phrase ${ }^{29}$. En ce sens, la parenthèse est le lieu privilégié de la parataxe, du mot glissé dans la phrase sans réellement y avoir été intégré. C'est pourquoi elle accueille plus facilement les signes de ponctuation isolés, sortes d'équivalents graphiques des attitudes non verbales de l'oral, qui, en tant que telles, ne sont que rarement considérées comme partie intégrante du système linguistique. Les parenthèses permettent aussi de naviguer dans le texte via les notes et les renvois qui s'intercalent dans le fil du discours tout en étant extérieures. Enfin, elles accueillent tout ce qui se superpose au texte sans en faire réellement partie comme les syntagmes nominaux sans préposition en fonction de déterminant du nom ou les adverbes à la portée sémantique limitée à un seul mot.

Enfin, si les parenthèses sont des signes de l'énonciation et les tirets des signes de phrase, cela explique sans doute pourquoi seules les premières sont aptes à recueillir les commentaires sur un discours autre. Le discours direct est en effet un phénomène linguistique par lequel un énonciateur introduit dans son discours un discours autre dont il n'assume pas la responsabilité énonciative. Ce discours est intégré tel quel au sein de la phrase qui l'accueille (voir Van Raemdonck, 2002). Il est généralement marqué typographiquement au moyen des guillemets ou de l'italique (voir François, 2006: 190-22I), qui en marquent les limites. Normalement, le retour à l'énonciateur principal est signalé typographiquement (fermeture des guillemets, retour aux caractères romains). On peut dès lors penser que les tirets, en tant que ponctuation de phrase, ne permettent pas le changement d'énonciateur, au contraire de la parenthèse qui, en tant que signe de l'énonciation, permet le retour au discours citant.

Le seul cas qui semble faire exception est celui de l'incise. L'incise fonctionne justement différemment. Tout d'abord, en termes de ponctuation, elle est encadrée de virgules, alors qu'il serait incongru d'encadrer son commentaire dans le discours de l'autre avec ce même signe. Ensuite, il est possible de l'analyser syntaxiquement comme remplissant une fonction dans l'énoncé cité (celle de complément de l'énonciation) et donc comme faisant partie intégrante de cet énoncé (voir Wilmet, 2oro: 671-673 et Van Raemdonck, 2002).

29. Cela explique sans doute l'intuition d'acceptabilité moindre de référence au contenu décroché hors de celui-ci avec les parenthèses, que nous soulevions en note i7. 


\section{Conclusion}

Si les parenthèses et les tirets marquent tous deux un phénomène clairement discursif, le décrochement énonciatif, notre corpus a montré que ces signes ne sont pas interchangeables. Certes, les tirets peuvent toujours être remplacés par des parenthèses, mais l'inverse n'est pas vrai.

Certains usages de la parenthèse semblent lui être propres, que ce soit au niveau pragmatique ou sémantique (commentaires sur un discours autre, marques de navigation au sein ou à l'extérieur du texte, signes de ponctuation isolés, dates de naissance et de mort), syntaxique (segments inférieurs au mot et au moins égaux à la phrase) ou typographique (signe double - vs signe simple -, possibilité d'accueillir des marques graphiques de la phrase).

Nous avons tenté de montrer que ces différences de fonctionnement peuvent s'expliquer par une différence en termes de place dans le système de la ponctuation. Selon le modèle que nous avons développé (François, 2006), les tirets sont des signes de phrase (dont la portée est strictement inférieure à celle-ci), alors que les parenthèses sont à proprement parler des signes de l'énonciation, c'est-à-dire des signes marquant des phénomènes énonciatifs à tous les niveaux linguistiques - des figures graphiques au texte.

Ceci explique pourquoi les parenthèses peuvent toujours se substituer aux tirets alors que l'inverse n'est pas vrai. Cela permet aussi de comprendre que les parenthèses doivent toujours être refermées (on ne peut pas deviner où s'arrête leur portée), alors que le tiret fermant n'est pas réalisé devant une ponctuation finale de phrase (puisque sa portée est inférieure à celle du point par exemple, ce dernier marque, en même temps que la fin de la phrase, la limite finale de la séquence ouverte par le tiret). C'est pour cette même raison que la parenthèse peut accueillir des phrases entières (avec leurs marques initiales et finales que sont respectivement la majuscule et le point) alors que le tiret ne le peut pas.

En allant plus loin, nous parvenons à montrer que les différences sémantiques trouvent leur explication dans ce même phénomène. Les parenthèses, en tant qu'elles ne sont pas attachées à un niveau de marquage linguistique, accueillent plus volontiers tout ce qui est étranger au texte lui-même (ponctuations isolées, renvois et notes, dates de naissance et de mort).

Notre hypothèse explique également les fonctionnements énonciatifs différents de nos signes. L'incise, complément de l'énonciation dans le discours direct, est intégrée à la syntaxe du discours cité (elle peut être encadrée de virgules): on peut donc la trouver marquée de tirets et de parenthèses. En revanche, lorsque l'énonciateur principal commente le discours cité, seules les parenthèses, véritables marques de l'énonciation, permettent le changement de voix.

Bien sûr, une étude de corpus est forcément limitée, si bien que certains usages nous ont sans doute échappé. Certains phénomènes que nous avons dit ne pas 
exister, peuvent peut-être se rencontrer. Nous pensons cependant que, si nous ne les avons pas rencontrés, c'est qu'ils sont rares et cela mérite sans doute déjà une explication... Nous espérons en ce sens offrir une base de réflexion intéressante, même si certains faits viennent contredire nos observations.

\section{Bibliographie}

Anis, J. 1988. L'écriture. Théories et descriptions. Avec la collaboration de J.-L. CHiss et C. Puech. Bruxelles: De Boeck-Wesmael.

СатасH, N. 1996. La ponctuation. Histoire et système. Paris: PUF.

DAhLET, V. 2003. Ponctuation et énonciation. Matoury (Guyane): Ibis rouge.

DoppaGne, A. 1998. La bonne ponctuation. Clarté, efficacité et précision de l'écrit. Paris - Bruxelles: Duculot.

Drillon, J. 1991. Traité de la ponctuation française. Paris: Gallimard.

DugAs, A. 2004. Guide de la ponctuation. Montréal: Éditions Logiques.

FrançoIs, G. 2006. Analyse et description des usages contemporains de la ponctuation française. Thèse de doctorat non publiée. Faculté de philosophie et de lettres, Université Libre de Bruxelles.

HaLkin, L. 1948. La technique de l'édition: conseils aux auteurs pour la préparation de leur copie et la correction des épreuves. Paris: Desclée de Brouwer.

IMPRIMERIE NATIONALE 2002. Lexique des règles typographiques en usage à l'imprimerie nationale. Paris: Imprimerie nationale.

Le Goffic, P. 1993. Grammaire de la phrase française. Paris: Hachette.

MOURAD, G. 1999. Analyse informatique des signes typographiques pour la segmentation de textes et l'extraction automatique de citations. Réalisation des applications informatiques: SegATex et CitaRE. Thèse de doctorat non publiée. Université de Paris-Sorbonne.

Pétillon-Boucheron, S. 2003. Les détours de la langue. Étude sur les parenthèses et le tiret double. Louvain - Paris: Peeters.

SerÇA, I. 20IO. Les coutures apparentes de la Recherche. Proust et la ponctuation. Paris: Champion.

Tournier, C. 1980. Histoire des idées sur la ponctuation des débuts de l'imprimerie à nos jours. Langue française $45: 28-40$.

VAN RAEMDONCK, D. 2002. Discours rapporté et frontières de phrase : l'épreuve de l'intégration syntaxique. Faits de langue 19: 171-178.

VÉDÉNINA, L. 1989. Pertinence linguistique de la présentation typographique. Paris: Peeters - SELAF.

WiLmet, M. 20ı. Grammaire critique du français. Bruxelles: Duculot. 